\title{
Percutaneous Mitral Valvuloplasty in a Patient With a Previous Transcatheter Aortic Valve Implantation
}

\author{
Xavier Millan ${ }^{\mathrm{a}, \mathrm{b}, \mathrm{c}}$, Dabit Arzamendi ${ }^{\mathrm{b}}$, Antonio Serra ${ }^{\mathrm{b}}$
}

\begin{abstract}
Successive percutaneous procedures are an emerging scenario in structural interventional cardiology. More experience is needed in this field but good outcomes already predict a promising future. We herein present a percutaneous mitral balloon valvuloplasty in a patient who previously received a transcatheter aortic valve implantation.
\end{abstract}

Keywords: Mitral ballon valvuloplasty; Valve disease; Transcathete aortic valve replacement; Interventional procedures

\section{Introduction}

With the progressive and successful development of percutaneous interventional techniques for valve diseases treatment, an increasing number of patients with rheumatic or degenerative valve disease will be referred to our institutions for successive procedures, representing a new scenario for interventional cardiology. We describe a percutaneous mitral balloon valvuloplasty in a patient with a previous transcatheter aortic valve implantation.

\section{Case Report}

A fragile 80 year-old woman with a history of hypertension,

\footnotetext{
Manuscript accepted for publication August 14, 2013

${ }^{a}$ Department of Medicine, Montreal Heart Institute, University of Montreal, Quebec, Canada

${ }^{\mathrm{b}}$ Department of Interventional Cardiology, Hospital de Sant Pau i la Santa Creu, Universitat Autonoma de Barcelona, Catalunya, Spain ${ }^{\mathrm{c}}$ Corresponding Author: Xavier Millan, Montreal Heart Institute, 5000 Belanger Est, Montréal H1T 1C8, Quebec, Canada.

Email: xavier.millan.alvarez@umontreal.ca

doi: http://dx.doi.org/10.4021/jmc1489w
}

dyslipidaemia and bronchial hyper reactivity was transferred to our hospital with suspected heart failure. She had a paroxystic atrial fibrillation and a femoral Transcatheter Aortic-Valve Implantation (TAVI) with a Core Valve\#26 was performed three years ago due to a severe degenerative aortic stenosis with dyspnea. After the TAVI her symptoms improved but she persisted with dyspnea (New York Heart Association functional class II - III). She was receiving warfarin, beta-blockers, ACE inhibitors, furosemide, statins, bronchodilators and analgesics.

She was admitted for progressive shortness of breath, orthopnea and daily episodes of paroxysmal nocturnal dyspnea. She denied dietary transgressions or treatment noncompliance. Blood pressure was 110/75 $\mathrm{mmHg}$; pulse was regular at $70 \mathrm{bpm}$, breathing rate $24 / \mathrm{min}$, oxygen saturation $89 \%$ (26\% oxygen). Clinical and radiologic exploration was consistent with acute heart failure, with a loud first heart sound and a holodiastolic apical rumbling murmur. The ECG showed sinus rhythm and a right bundle branch block without signs of ischemia. Blood tests were remarkable for mild anaemia (Hb: $10.2 \mathrm{~g} / \mathrm{dL}, \mathrm{MCV}: 89.4 \mathrm{fL}$ ), pharmacological anticoagulation (INR: 3.16 ) and acute renal failure (Creatinine: $1.6 \mathrm{mg} / \mathrm{dL}$, glomerular filtration: $33 \mathrm{~mL} / \mathrm{min}$ ).

Intravenous diuretics were initiated, ACE inhibitors and beta-blockers were initially stopped and the patient improved her symptoms but persisted with dyspnea on minimal exertion and need of nocturnal oxygen.

An echocardiogram showed a non-dilated, hypertrophic left ventricle with normal ejection fraction. Both atria were dilated and the aortic prosthesis was normally functioning. The mitral ring was intensively calcified, with severe mitral stenosis. A $0.8 \mathrm{~cm}^{2}$ valve area was estimated, without significant regurgitation [1]. There was severe tricuspid regurgitation with an important pulmonary hypertension. There was no evidence of atrial thrombus.

The case was presented in the Heart Team for mitral valve replacement but it was dismissed for high-surgical risk [2] (age, intense mitral ring calcification and lack of experience with a patient with a previous Core Valve TAVI). After discussing the few management options with the patient and her family, a palliative percutaneous mitral balloon valvuloplasty was accepted $[3,4]$. The procedure was performed 


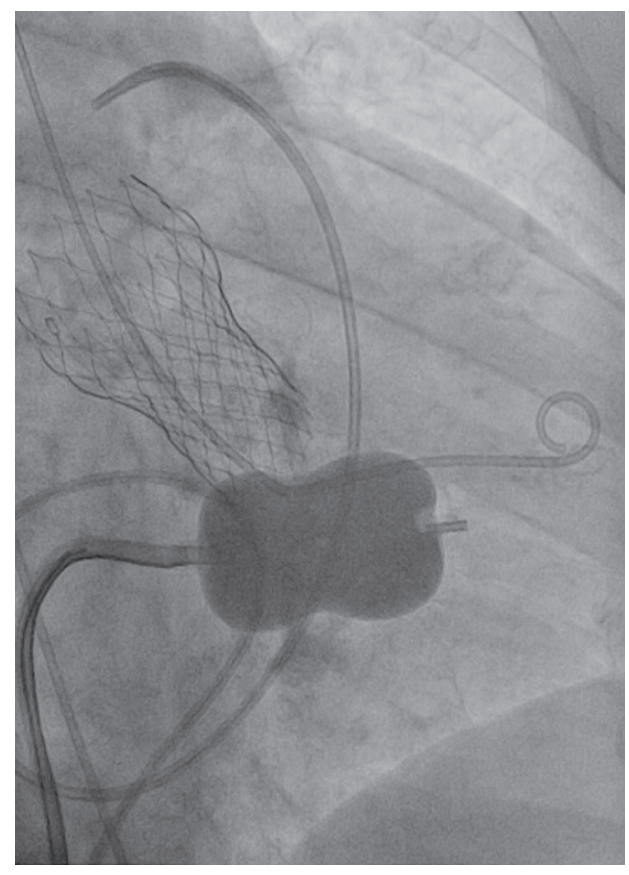

Figure 1. Fluoroscopy image with mitral balloon inflation and previous aortic CoreValve.

with the patient under light sedation. A transesophageal echocardiography discarded atrial thrombosis and guided the transeptal catheterization with the standard Brockenbrough technique $[5,6]$, via a femoral approach with an $8 \mathrm{~F}$ sheath in the vein and a $5 \mathrm{~F}$ sheath in the artery. The interatrial puncture was successful and the Mullins catheter could be located in the left atrium through a coiled-tip guide wire. The pigtail was positioned in the left ventricle and a medium diastolic gradient between left atrium and ventricle of $13 \mathrm{mmHg}$ was registered, estimating an initial mitral valve area of $0.8 \mathrm{~cm}^{2}$ (cardiac output of $3.2 \mathrm{~L} / \mathrm{min}$ ).

$7000 \mathrm{U}$ of i.v. heparin were administered to reduce the risk of a thromboembolic event during the manipulation of wires and catheters in the left atrium, and activated clotting time was strictly monitored to maintain appropriate levels of anticoagulation during the procedure [7].

Afterwards, the interatrial septum was dilated with a $14 \mathrm{~F}$ dilator and the Mullins catheter was positioned in the left atrium forming a loop with its tip facing toward the mitral valve orifice. With the right anterior oblique view, a $28 \mathrm{~mm}$ Inoue balloon was progressed and crossed through the mitral valve. After assuring there was no entrapment in the subvalvular apparatus[7], consecutive balloon inflations were done, increasing the balloon diameter progressively from $24 \mathrm{~mm}$ to $28 \mathrm{~mm}$ (Fig. 1) and measuring the transvalvular gradient after each inflation, to assess the valve area and to avoid an eventual worsening of a mitral regurgitation [7]. Finally, after five balloon inflations, a significant reduction of transvalvular gradient was noticed (from $13 \mathrm{mmHg}$ to $8 \mathrm{mmHg}$ ), with a post-procedure calculated mitral area of $1.2 \mathrm{~cm}^{2}$.

After the procedure, the patient showed an important clinical improvement. Oxygen could be withdrawn and renal function normalized. And echocardiogram showed a mitral valve area by planimetry of $1.2 \mathrm{~cm}^{2}$ Beta-blockers, ACE inhibitors and warfarin could be reintroduced and she could be discharged five days after the mitral valvuloplasty, without any complication. One year later, she maintains a NYHA II functional class, without requiring any hospital admission.

\section{Discussion}

In current practice, surgery for mitral stenosis is mostly valve replacement $(95 \%)$ as a result of unfavourable valve characteristics for valve repair. Percutaneous mitral valvuloplasty is the procedure of choice when, as in our case, surgery is contraindicated and symptoms persist despite optimal medical treatment [8]. The most striking feature of our case is the fact that the patient had previously received a transcatheter aortic valve implantation.

In conclusion, TAVI treated patients may develop disease in other valves, particularly mitral stenosis in cases of rheumatic heart disease or, more frequently, in degenerative aetiology. The gradual aging of population and the development of less invasive techniques may increase the performance of successive percutaneous procedures in structural interventional cardiology.

\section{References}

1. Sanati HR, Zahedmehr A, Shakerian F, Bakhshandeh H, Firoozi A, Kiani R, Sadeghpour A, et al. Percutaneous mitral valvuloplasty using echocardiographic intercommissural diameter as reference for balloon sizing: a randomized controlled trial. Clin Cardiol. 2012;35(12):749754.

2. Holloway S, Silver RS. 2010. Percutaneous Valve Commissurotomy, Repair, and Replacement. In: Watson S, Gorski KA. Invasive Cardiology: A Manual for Cath Lab Personnel 3d ed. Inc 271-298.

3. Nobuyoshi M, Arita T, Shirai S, Hamasaki N, Yokoi H, Iwabuchi M, Yasumoto H, et al. Percutaneous balloon mitral valvuloplasty: a review. Circulation. 2009;119(8):e211-219.

4. Palacios IF. 2010. Percutaneous Mitral Balloon Valvuloplasty for Patients with Rheumatic Mitral Stenosis: Immediate and Long-Term Follow-up Results. In: Herrmann HC. Interventional Cardiology: percutaneous noncoronary intervention. New Jersey. Inc. p 3-28.

5. Nguyen TN, Ischinger T, Nhan VT, Kumar R. 2009. Balloon Angioplasty. In: Nguyen TN, Colombo A, Hu D, Grines CL, Saito S, editors. Practical Handbook of 
Advanced Interventional Cardiology: Tips and Tricks 3d ed. Victoria, Inc. p 85-95.

6. Palacios IF, Arzamendi D. Structural heart intervention. Beyond transcatheter valve therapy. Rev Esp Cardiol (Engl Ed). 2012;65(5):405-413.

7. Vahanian A, Bassand JP, Boudjemline Y, Cribier A. 2007. Complications of percutaneous valve interven- tions. In: Eeckhout E, Lerman A, Carlier S, Kern M, editors. Handbook of Complications during Percutaneous Cardiovascular Interventions. London. Inc. p 269-286.

8. Feldman T. 2006. Percutaneous Therapies for Valvular Heart Disease. In: Baim DS editor. Grossman's Cardiac Catheterization, Angiography, \& Intervention 7th ed. Inc. p 544-562. 